\title{
Variational methods for steady-state Darcy/Fick flow in swollen and poroelastic solids
}

\author{
TOMÁŠ ROUBÍČEK \\ Mathematical Institute, Charles University, Sokolovská 83, CZ-186 75 Praha 8, Czech Republic, \\ and \\ Institute of Thermomechanics, CAS, Dolejškova 5, CZ-182 00 Praha 8, Czech Republic.
}

\begin{abstract}
Existence of steady states in elastic media at small strains with diffusion of a solvent or fluid due to Fick's or Darcy's laws is proved by combining usage of variational methods inspired from static situations with Schauder's fixed-point arguments. In the plain variant, the problem consists in the force equilibrium coupled with the continuity equation, and the underlying operator is non-potential and non-pseudomonotone so that conventional methods are not applicable. In advanced variants, electrically-charged multi-component flows through an electrically charged elastic solid are treated, employing critical points of the saddle-point type. Eventually, anisothermal variants involving heat-transfer equation are treated, too.
\end{abstract}

AMS Subject Classification: 35Q74, 49S05, 74F10, 74F15, 76S05, 78M30, 80A17.

\section{Introduction}

Some elastic materials allow for a penetration of very small atoms into a solid atomic grid in crystalline metals or into spaces between big macromolecules of polymers. In the former case, the interstitial solute is hydrogen and such metals then undergo a so-called metal-hydride phase transformation as schematically depicted in Fig. 1-left, see e.g. $[6,7,11]$. The latter mentioned mechanism occurs in polymers allowing for a diffusion of a specific solvent causing unpacking of macromolecules as schematically depicted in Fig. 1-middle. In both cases, the solvent which diffuses thorough the elastic body influences the volume considerably (sometimes by tens of percents), which is referred to as a swelling. In turn, this swelling influences stress/strain distribution and therefore also the diffusion process itself. The diffusion is driven rather by the concentration gradient, which is referred to as a Fick law.

Other, microscopically different mechanism occurs in macroscopically solid materials that posses various pores or voids which are mutually connected and which allows for some fluids (sometimes referred to as a diffusant) to flow thorough the solid. It is manifested macroscopically as a homogeneous mixture of a solid elastic body and fluid which diffuses throughout the volume as schematically depicted in Fig. 1-right. Examples are poroelastic rocks or porous polymers filled with water (and in the latter case possibly also with ionized hydrogen, i.e. protons, while the poroelastic polymer itself is negatively charged by fixed dopands as used in polymer-electrolyte fuel cells [8]). Interaction of solids with diffusants may be manifested by squeezing. The diffusion is driven rather by the pressure gradient, which is referred to as Darcy's law.

The solid-diffusant interaction is surely a complicated multi-scale problem and a big amount of phenomenology is usually applied to build a simplified model. A wide menagerie of models can thus be obtained, cf. [9] for a survey. Typically, small velocity of the diffusant is assumed. In this paper, we additionally assume small strains.

In order to explain our ideas on a simple steady-state problem, we introduce a material model in which elasticity depends on an internal variable $c$ denoting a concentration of a diffusant or fluid. Concretely, in the simplest variant we will be concerned with the following boundary-value problem:

$$
\begin{array}{ll}
\operatorname{div} \sigma+f=0 & \text { with } \quad \sigma=\partial_{e} \varphi(e(u), c) \\
\operatorname{div}(\mathbb{M}(c) \nabla \mu)=0 & \text { with } \quad \mu=\partial_{c} \varphi(e(u), c)
\end{array}
$$

to be solved on a bounded Lipschitz domain $\Omega \subset \mathbb{R}^{d}$. Later we will also investigate various generalization towards thermodynamics or coupling with electric field. The system (1) should be completed 


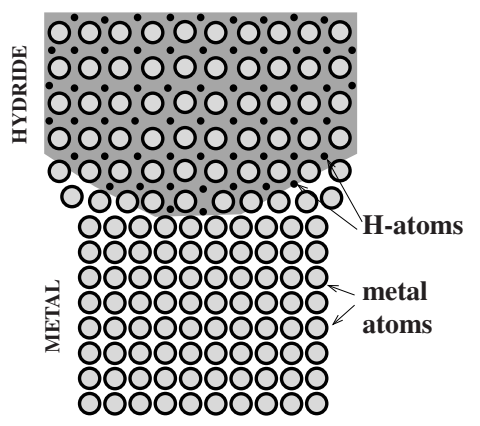

materials undergoing swelling

(Fick flow)

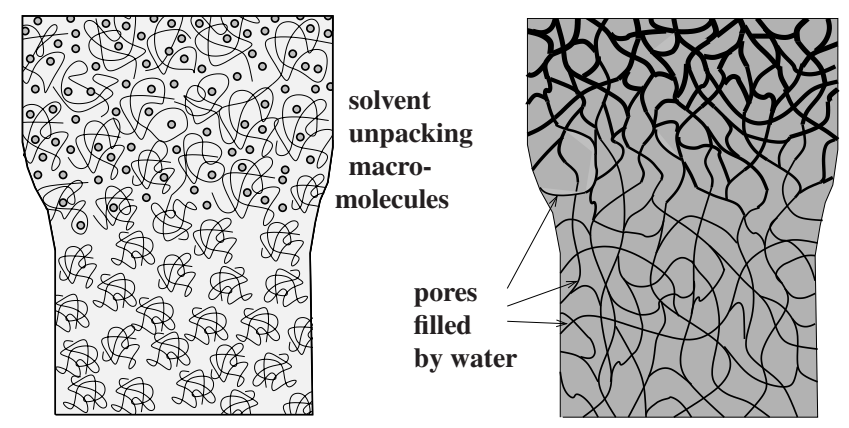

porous materials

(Darcy flow)

Figure 1: Various underlying mechanisms coupling diffusion with volume expansion, ranging from an atomic via a molecular to a mesoscopical level:

Left: hydrogen diffusing inside a metallic atomic grid (metal/hydride transformation).

Middle: macromolecules in polymers unpacked by a diffusing solvent.

Right: water flowing through pores of a (poro)elastic rock or a concrete

by suitable boundary conditions, e.g.

$$
\begin{aligned}
& u=0 \text { on } \Gamma_{\mathrm{D}}, \quad \sigma \vec{n}=g \text { on } \Gamma_{\mathrm{N}}, \\
& \mathbb{M}(c) \nabla \mu \cdot \vec{n}+\alpha \mu=\alpha \mu_{\text {ext }} \text { on } \Gamma,
\end{aligned}
$$

where $\Gamma$ denotes the boundary of $\Omega$ divided into the parts $\Gamma_{\mathrm{D}}$ and $\Gamma_{\mathrm{N}}$.

One should distinguish between the general steady-state (sometimes also called stationary) situations and purely static situation (in the sense of steady states in thermodynamical equilibrium). The former one means that all fields including the specific dissipation rates are independent of time, while the latter means in addition that the dissipation rates are zero (which here means that all transportation processes vanish).

The plan of this paper is as follows: In Section 2, we start with examples of free energies $\varphi$ typically arising in problems from continuum mechanics of poroelastic or swelling-exhibiting materials. The static case enjoys a full variational structure, which is presented in Section 3. It can be exploited for general steady-state problems where it is combined with the Schauder fixed point technique, which is scrutinized in Section 4. The steady-state problems (1)-(2) suggest interesting thermodynamically consistent anisothermal augmentation by a heat-transfer equation. This again combines variational technique for auxiliary static-like problems with Schauder's fixed point arguments, as presented in Section 5. In each Sections 3-5, we also consider a (possibly electrically charged) multi-component generalization which leads to a necessity of considering critical points of a saddle-point-type variational problems instead of mere minimizers. Various remarks close this paper in Section 6, addressing in particular uniqueness issue, electroneutrality limit, or a general evolutionary thermodynamical context.

\section{Convex free energies leading to Fick or Darcy flows}

With a certain simplification, one may say that two basic alternatives in the isotropic continuum are that either the strain is directly influenced by a concentration $c$ of the solvent which diffuses through the material or the solvent has its own pressure which influences the overall stress and eventually indirectly the strain too. These two options microscopically reflect that the solvent does not directly contribute to the pressure but can change the reference configuration by influencing the atomic grid (as in the mentioned metal-hydride transformation) or the the solvent flows though pores under its pressure and, from the macroscopical viewpoint, the resulted porous solid is phenomenologically homogenized. 
The simplest form of the free energy in the former option is

$$
\varphi(e, c)=\frac{1}{2} \mathbb{C} e_{\mathrm{el}}: e_{\mathrm{el}}+\kappa\left(c\left(\ln \frac{c}{c_{\mathrm{eq}}}-1\right)+\delta_{(0, \infty)}(c)\right) \text { with } e_{\mathrm{el}}:=e-E c+E c_{\mathrm{eq}},
$$

with $\mathbb{C} \in \mathbb{R}^{d \times d \times d \times d}$ a symmetric positive definite 4rt-order elasticity tensor, $\kappa \geq 0$ a coefficient weighting chemical versus mechanical effects, $E \in \mathbb{R}_{\mathrm{sym}}^{d \times d}$ a matrix of the swelling coefficients (which, in isotropic materials, is an identity matrix up to a coefficient) and with $c_{\mathrm{eq}}$ an equilibrium concentration minimizing $\varphi(0, \cdot)$. Moreover, $\delta_{S}(c)$ denotes the indicator function, i.e. $\delta_{S}(c)=0$ if $c \in S$ while $\delta_{S}(c)=\infty$ otherwise. This yields the stress

$$
\sigma=\partial_{e} \varphi(e, c)=\mathbb{C} e_{\mathrm{el}}=\mathbb{C}\left(e-E c+E c_{\mathrm{eq}}\right)
$$

and the chemical potential $\mu=\partial_{c} \varphi(e, c)=\kappa \ln c-E: \sigma$ for $c>0$, or, taking into account that $\varphi(e, \cdot): \mathbb{R} \rightarrow \mathbb{R} \cup\{\infty\}$ is a proper convex function which is nonsmooth, rather

$$
\mu \in \partial_{c} \varphi(e, c)=\left\{\begin{array}{cl}
\left\{\kappa \ln \left(c / c_{\mathrm{eq}}\right)-E: \sigma\right\} & \text { if } c>0 \\
\emptyset & \text { if } c \leq 0
\end{array}\right.
$$

note that the last term $E: \sigma$ is the pressure. Also note that $\lim _{c \rightarrow 0+} \partial_{c} \varphi(e, c)=-\infty$, which causes that indeed $\partial_{c} \varphi(e, 0)=\emptyset$, cf. also [10, Fig. 11].

The latter option relies on the idea that the diffusant (fluid) fully occupies the pores (i.e. so-called saturated flow) whose volume is proportional to $\operatorname{tr} e(u)$ through a coefficient $\beta>0$ and its pressure is

$$
p_{\text {fld }}=M\left(\beta \operatorname{tr} e(u)-c+c_{\text {eq }}\right)
$$

with $M$ allowing an interpretation as the compressibility modulus of the fluid; actually, $M$ and $\beta$ are the so-called Biot modulus and the Biot coefficient used in conventional models of porous media [3]. The (positive) parameter $c_{\text {eq }}$ denotes the equilibrium concentration and is considered here fixed, being related with the porosity of the material which is here considered as a fixed material property. This pressure is then summed up with the stress $\sigma_{\mathrm{el}}$ in the elastic solid. In isotropic materials, the total stress is then $\sigma=\sigma_{\mathrm{el}}+\beta p_{\mathrm{fld}} \mathbb{I}$. In such simplest variant, it leads to the potential

$$
\varphi(e, c)=\frac{1}{2} \mathbb{C} e: e+\frac{1}{2} M\left(\beta \operatorname{tr} e-c+c_{\mathrm{eq}}\right)^{2}+\kappa\left(c\left(\ln \frac{c}{c_{\mathrm{eq}}}-1\right)+\delta_{(0, \infty)}(c)\right),
$$

which yields the stress and the chemical potential

$$
\begin{aligned}
& \sigma=\partial_{e} \varphi(e, c)=\mathbb{C} e+\beta M\left(\beta \operatorname{tr} e-c+c_{\mathrm{eq}}\right) \mathbb{I} \text { and } \\
& \mu \in \partial_{c} \varphi(e, c)=\left\{\begin{array}{cl}
\left\{M\left(c-\beta \operatorname{tr} e-c_{\mathrm{eq}}\right)+\kappa \ln \left(c / c_{\mathrm{eq}}\right)\right\} & \text { if } c>0, \\
\emptyset & \text { if } c \leq 0,
\end{array}\right.
\end{aligned}
$$

the emptyness of the subdifferential for $c \leq 0$ being analogous to (3c).

Then, choosing still a standard ansatz $\bar{M}(c)=c \mathbb{M}_{0}$, the flux $j=-\mathbb{M}(c) \nabla \mu$ turns into

$$
j=\underbrace{-c \mathbb{M}_{0} \nabla p}_{\begin{array}{c}
\text { Darcy } \\
\text { law }
\end{array}} \underbrace{-\mathbb{M}_{0} \kappa \nabla c}_{\begin{array}{c}
\text { Fick } \\
\text { law }
\end{array}} \quad \text { with } p= \begin{cases}E: \mathbb{C}\left(e-E c+E c_{\text {eq }}\right) & \text { in case (3), } \\
M\left(\beta \operatorname{tr} e-c+c_{\text {eq }}\right) & \text { in case (5) }\end{cases}
$$

provided $c>0$. Depending on $\kappa$, either Darcy's mechanism or the Fick's one may dominate. Note also that $|p|=\mathscr{O}(|E|)$ in the case (3). An interesting phenomenon is that the equilibrium concentration $c_{\mathrm{eq}}$ does not influence $\nabla \mu$ and can influence the solution only through the boundary conditions (2). The mass-conservation equation (8b) reveals that the pressure gradient $\nabla p$ is needed and it 
also reveals a certain "optical" difficulty because there is no obvious estimate on $\nabla p$. Indeed, in the evolution variant (except simple linearized problems like (5a) for $\kappa=0$ and $\mathbb{M}(\cdot)$ constant, like considered in fact in [13]), a certain "regularization" of the problem seems to be necessary, by introducing a suitable phase field and its gradient as in [11], or a gradient of $c$ leading to the Cahn-Hilliard "capillarity-like" model, or a gradient of $e(u)$ leading to a 2 nd-grade nonsimple material concept.

Yet, the standard definition of a weak solution to the boundary-value problem (1)-(2) avoids explicit occurrence of $\nabla p$, and it indeed works if $\mathbb{M}$ is constant; note that the fixed-point argument used in the proofs of all "non-static" Propositions 4.1-5.2 becomes rather trivial because the distribution of the chemical potential $\mu$ is then fully determined by $\mu_{\text {ext }}$ in the boundary condition $(2 b)$. However, quite surprisingly, the non-static steady-state models allow for a lot of results without any regularization even when $\mathbb{M}$ depends on $c$; cf. also Remark 6.2 below.

The following standard notation will be used: $L^{p}$ will stand for the Lebesgue spaces of measurable functions whose $p$-power is integrable and $W^{1, p}$ for Sobolev spaces whose distributional derivatives are in $L^{p}$-spaces. For $p=2$, we abbreviate $H^{1}=W^{1,2}$. Moreover, we use the standard notation $p^{\prime}=p /(p-1)$, and $p^{*}$ for the Sobolev exponent $p^{*}=p d /(d-p)$ for $p<d$ while $p^{*}<\infty$ for $p=d$ and $p^{*}=\infty$ for $p>d$, and the "trace exponent" $p^{\sharp}$ defined as $p^{\sharp}=(p d-p) /(d-p)$ for $p<d$ while $p^{\sharp}<\infty$ for $p=d$ and $p^{\sharp}=\infty$ for $p>d$. Thus, e.g., $W^{1, p}(\Omega) \subset L^{p^{*}}(\Omega)$ or $L^{p^{* \prime}}(\Omega) \subset W^{1, p}(\Omega)^{*}$. In the vectorial case, we will write $L^{p}\left(\Omega ; \mathbb{R}^{n}\right) \cong L^{p}(\Omega)^{n}$ and $W^{1, p}\left(\Omega ; \mathbb{R}^{n}\right) \cong W^{1, p}(\Omega)^{n}$. We will also use the notation $H_{\mathrm{D}}^{1}\left(\Omega ; \mathbb{R}^{d}\right):=\left\{u \in H^{1}\left(\Omega ; \mathbb{R}^{d}\right) ;\left.u\right|_{\Gamma_{\mathrm{D}}}=0\right\}$. Eventually, "." or ":" denotes the scalar products of vectors or matrices, respectively.

Moreover, in what follows we will use the standard notation " $\partial$ " either for the partial derivative or a (partial) convex subdifferential. Without restricting generality towards applications of our interest, we assume $\varphi$ nonsmooth in terms of $c$ only so that $\partial_{e} \varphi$ will be single-valued. Our variational methods will need coercivity of the involved convex or convex/concave functionals but not necessarily their controlled growth, so the related Euler-Lagrange equations are to be understood in a variational rather than a conventional weak sense. Avoiding the growth conditions is one of the benefits of the variational approach to this problem.

\section{Problems in thermodynamical equilibrium}

Let us now focus on special steady-state problems where the dissipation rate is not only constant in time but just zero. Then also temperature is not influenced by the mechanical/diffusion part and we can ignore the heat transfer. As we already said, such problems are called static. The diffusion is related with the dissipation rate (and entropy and heat production rate), namely that the overall dissipation (or also the heat-production) rate is $\int_{\Omega} \mathbb{M} \nabla \mu \cdot \nabla \mu \mathrm{d} x$, cf. also to (40) below. This implies $\nabla \mu$ everywhere on $\Omega$, and in particular also the boundary flux $j=-\mathbb{M} \nabla \mu$ is to be zero. When assuming $\Omega$ connected in such static case, $\nabla \mu=0$ on $\Omega$ leads to that $\mu$ is constant. Let us denote this constant by $\bar{\mu}$.

Therefore, solvability of such problem essentially requires either the system to be in equilibrium with the external environment or to be isolated. The former option is rather trivial: assuming $\mu_{\text {ext }}=\bar{\mu}$ in (2) with a given constant $\bar{\mu}$ and $\alpha>0$, it fixes $\mu=\bar{\mu}$ and then one can eliminate $c$. From $\bar{\mu}=\partial_{c} \varphi(e, c)$, we can then find the concentration $c=\left[\partial_{c} \varphi(e, \cdot)\right]^{-1}(\bar{\mu})$ as a function of $e$. If $\varphi(e, \cdot)$ is convex, we can even write a bit more specifically

$$
c=\partial_{\mu} \varphi^{*}(e, \bar{\mu})
$$

with $\varphi^{*}(e, \cdot)$ denoting the convex conjugate function of $\varphi(e, \cdot)$. Note that, even if $\varphi(e, \cdot)$ is not smooth as in the examples in Section $2, \varphi^{*}(e, \cdot)$ is indeed single-valued if the natural requirement $\partial_{c c}^{2} \varphi>0$ holds. The concentration $c$ can thus be completely eliminated.

The latter option (i.e. the boundary permeability coefficient $\alpha=0$ ) is more interesting. When the profile $e=e(u(x))$ is known, the overall amount of solvent $\int_{\Omega} c \mathrm{~d} x=C_{\text {total }}$ depends monotonically on $\bar{\mu}$ due to (6), which allows us to specify $\bar{\mu}$ if $C_{\text {total }}$ is given. Yet, the displacement $u$ is a part of solution itself so that, unfortunately, it does not seem possible to fix $\bar{\mu}$ just from knowing $C_{\text {total }}$. In 
this isolated situation, it is natural to prescribe the total amount of diffusant

$$
\int_{\Omega} c \mathrm{~d} x=C_{\text {total }} \quad \text { with } \quad C_{\text {total }} \geq 0 \text { given. }
$$

Interestingly, this special steady-state (=static) case enjoys a full variational structure at least in the sense that some (if not all) solutions can be obtained by such way as critical points. The general steady-state system (1) then modifies to

$$
\begin{aligned}
& \operatorname{div} \partial_{e} \varphi(e(u), c)+f=0, \\
& \partial_{c} \varphi(e(u), c) \ni \bar{\mu}=\text { some constant, }
\end{aligned}
$$

to be coupled with (7) and with the boundary conditions (2a). Let us recall that $\varphi(e, \cdot)$ is allowed to be nonsmooth so that $\partial_{c} \varphi$ maybe set-valued so that $(8 \mathrm{~b})$ is an inclusion rather than equation. More specifically, the mentioned variational structure consists in the following constrained minimization problem is of a certain relevance:

$$
\left.\begin{array}{ll}
\text { Minimize } & (u, c) \mapsto \int_{\Omega} \varphi(e(u), c)-f \cdot u \mathrm{~d} x-\int_{\Gamma_{\mathrm{N}}} g \cdot u \mathrm{~d} S \\
\text { subject to } & \int_{\Omega} c \mathrm{~d} x=C_{\text {total }}, \quad u \in H_{\mathrm{D}}^{1}\left(\Omega ; \mathbb{R}^{d}\right), \quad c \in L^{1}(\Omega) .
\end{array}\right\}
$$

Proposition 3.1 (Existence of static solutions). Let $\varphi: \mathbb{R}_{\mathrm{sym}}^{d \times d} \times \mathbb{R} \rightarrow \mathbb{R} \cup\{\infty\}$ be convex, lower semicontinuous, and coercive in the sense that $\varphi(e, c) \geq \epsilon|e|^{2}+\epsilon|c|^{1+\epsilon}$ for some $\epsilon>0$, meas $_{d-1}\left(\Gamma_{\mathrm{D}}\right)>0$, $f \in L^{2^{* \prime}}\left(\Omega ; \mathbb{R}^{d}\right)$, and $g \in L^{2^{\sharp}}\left(\Gamma_{\mathrm{N}} ; \mathbb{R}^{d}\right)$. Moreover, let the body be isolated (i.e. $\alpha=0$ ) and the overall content $C_{\text {total }}$ be given, assuming $\varphi\left(0, C_{\text {total }} /\right.$ meas $\left._{d}(\Omega)\right)<\infty$. Then the boundary-value problem (8) with boundary conditions (2a) and with the side condition (7) possesses at least one variational solution $(u, c) \in H_{\mathrm{D}}^{1}\left(\Omega ; \mathbb{R}^{d}\right) \times L^{1+\epsilon}(\Omega)$ whose chemical potential is constant over $\Omega$ in the sense that there exists a constant $\bar{\mu}$ such that $\int_{\Omega} \varphi(e(u), c)-\bar{\mu} c \mathrm{~d} x \leq \int_{\Omega} \varphi(e(u), \tilde{c})-\bar{\mu} \tilde{c} \mathrm{~d} x$ for all $\tilde{c} \in C(\Omega)$, in particular (8b) holds a.e. on $\Omega$ if $\partial_{c} \varphi(e(u), c)$ exists.

Proof. We use the direct method for minimization of the convex coercive functional subject to the affine constraint (9). Note that the assumption $\varphi\left(0, C_{\text {total }} / \operatorname{meas}_{d}(\Omega)\right)<\infty$ guarantees that this problem is feasible, i.e. its admissible set contains at least one pair $(u, c):=\left(0, C_{\text {total }} /\right.$ meas $\left._{d}(\Omega)\right)$. We thus obtain a minimizer $(u, c) \in H_{\mathrm{D}}^{1}\left(\Omega ; \mathbb{R}^{d}\right) \times L^{1+\epsilon}(\Omega)$.

As the functional in (9) is convex, lower semicontinuous, and the constraint is affine, introducing the Lagrange multiplier $\bar{\mu}$ to the scalar-valued constraint (7) which is involved in (9), this problem is equivalent to finding a critical point of the functional (a so-called Lagrangian):

$$
(u, c, \bar{\mu}) \mapsto J(u, c, \bar{\mu}):=\int_{\Omega} \varphi(e(u), c)-f \cdot u-\bar{\mu} c \mathrm{~d} x-\int_{\Gamma_{\mathrm{N}}} g \cdot u \mathrm{~d} S+C_{\text {total }} \bar{\mu} .
$$

If this functional is smooth, from putting the Gâteaux derivative with respect to $c$ zero, we can read that $\partial_{c} \varphi(e(u), c)=\bar{\mu}$ on $\Omega$, i.e. the chemical potential is constant. In a general nonsmooth case, by disintegration of the condition $\partial_{c} J(u, c, \bar{\mu}) \ni 0$ we obtain the inclusion (8b) a.e. on $\Omega$. From putting $\partial_{\bar{\mu}} J(u, c, \bar{\mu})=0$, we can read that $\int_{\Omega}-c \mathrm{~d} x+C_{\text {total }}=0$, i.e. that the affine constraint (7) is satisfied. Eventually, if the Gâteaux derivative $\partial_{u} J(u, c, \bar{\mu})$ exists, it must be zero, and we can read the equilibrium equation (8a) with the boundary conditions (2a) in the weak formulation. In a general case, (8a)-(2a) holds formally in a variational sense, i.e. the solution $u$ is considered as a minimizer of $J(\cdot, c, \bar{\mu})$.

The above proof reveals the role of the scalar $\bar{\mu}$ as the Lagrange multiplier to the scalar constraint $\int_{\Omega} c \mathrm{~d} x=C_{\text {total }}$, and it is a vital part of the solution. This is consistent with the above observation that, if the mechanical part of the solution $u$ is fixed, then $\bar{\mu}$ is determined by $C_{\text {total }}$.

If $\varphi$ is strictly convex (as e.g. in the examples from Sect.2) the minimization problem (9) has a unique solution, although the relation to solutions of the static problem in question may be more delicate, cf. Remark 6.1 below. 
An interesting and useful generalization of the above basic scenario is towards a multi-component fluid with $N \geq 2$ components which can even be electrically charged with specific charges $z=$ $\left(z_{1}, \ldots, z_{N}\right)$. Also the elastic medium can charged by some dopands with the specific charge $z_{\mathrm{DOP}}$. In the static problems there are no chemical reactions. The scalar-valued chemical potential is now to be replaced by an $\mathbb{R}^{N}$-valued electro-chemical potential

$$
\mu=\partial_{c} \varphi(e, c)+z \phi
$$

with $\phi$ the electrostatic potential. In static problems, again $\mu$ constant. The system (8) then augments to

$$
\begin{array}{ll}
\operatorname{div} \partial_{e} \varphi(e(u), c)+f=z_{\mathrm{DOP}} \nabla \phi & \text { on } \Omega, \\
\partial_{c} \varphi(e, c)+z \phi \ni \mu=\text { some constant } & \text { on } \Omega, \\
\operatorname{div}(\varepsilon \nabla \phi)+z \cdot c=z_{\mathrm{DOP}}+\operatorname{div}\left(z_{\mathrm{DOP}} u\right) & \text { on } \mathbb{R}^{d}, \\
\int_{\Omega} c \mathrm{~d} x=C_{\text {total }}=\text { a given constant } \in \mathbb{R}^{N} . &
\end{array}
$$

The right-hand side $z_{\mathrm{DOP}} \nabla \phi$ in (11a) is the Lorenz force acting on a charged elastic solid in the electrostatic field. In (11c), $\varepsilon=\varepsilon(x)>0$ denotes the permittivity and the equation (11c) itself is the rest of the full Maxwell system if all evolution and magnetic effects are neglected. Note that (11c) is to be solved on the whole universe with the natural "boundary" condition $\phi(\infty)=0$, assuming naturally that $z, c$, and $z_{\mathrm{DOP}}$ are extended on $\mathbb{R}^{d} \backslash \Omega$ by zero. Actually, the physical units are fixed for notational simplicity in such a way that the Faraday constant (which should multiply the charges in (11c)) equals 1 .

It is interesting that the underlying potential is not convex and, instead of a minimizer as in (9), we are now to seek a more general critical point, namely a saddle point solving the variational problem:

$$
\left.\begin{array}{c}
\operatorname{Min} / \max (u, c, \phi) \mapsto \int_{\Omega}\left(\varphi(e(u), c)+\left(z \cdot c-z_{\mathrm{DOP}}\right) \phi+z_{\mathrm{DOP}} \nabla \phi \cdot u\right. \\
-f \cdot u) \mathrm{d} x-\int_{\mathbb{R}^{d}} \frac{\varepsilon}{2}|\nabla \phi|^{2} \mathrm{~d} x-\int_{\Gamma_{\mathrm{N}}} g \cdot u \mathrm{~d} S,
\end{array}\right\}
$$

This convex/concave structure is sometimes referred under the name of electrostatic Lagrangian [8, Sect.3.2] and is consistent with a convex structure of the internal energy, cf. the argumentation in Remark 6.3 or (40)-(41) below.

Proposition 3.2. Let $\varphi: \mathbb{R}_{\mathrm{sym}}^{d \times d} \times \mathbb{R}^{N} \rightarrow \mathbb{R} \cup\{\infty\}$ and $\Gamma_{\mathrm{D}}$, $f$, and $g$ be qualified as in Proposition 3.1, $z_{\mathrm{DOP}} \in L^{\infty}(\Omega), z \in L^{\infty}\left(\Omega ; \mathbb{R}^{N}\right)$, and $\varepsilon \in L^{\infty}\left(\mathbb{R}^{d}\right)$ have a positive infimum. Moreover, let again $\alpha=$ 0 and $C_{\text {total }} \in \mathbb{R}^{N}$ be given so that $\varphi\left(0, C_{\text {total }} /\right.$ meas $\left._{d}(\Omega)\right)<\infty$. Then the boundary-value problem (11) with boundary conditions (2a) possesses at least one variational solution $(u, c) \in H_{\mathrm{D}}^{1}\left(\Omega ; \mathbb{R}^{d}\right) \times$ $L^{1+\epsilon}\left(\Omega ; \mathbb{R}^{N}\right)$ whose $\mathbb{R}^{N}$-valued electrochemical potential is constant over $\Omega$ in the sense that there exists a constant $\bar{\mu} \in \partial_{c} \varphi(e(u), c)+z \phi$ on $\Omega$ in the variational sense like in Proposition 3.1.

Proof. Existence of a saddle point in this problem is to be seen by the classical (Banach-space-valued extension [4] of) von Neumann theorem [16] (see also [17, Ch.49]) as well as that it yields some solution to the system (11) with $\mu$ being the (vector-valued) Lagrange multiplier to the constraint in (12). The assumption $\varphi\left(0, C_{\text {total }} /\right.$ meas $\left._{d}(\Omega)\right)<\infty$ again guarantees the feasibility of (12).

\section{General steady-state problems}

The peculiarity behind the general non-static steady-state problem (1) is that it mixes stored energy and the dissipation energy, cf. (40) below. Thus one should not expect a simple variational structure 
which is usual in problems governed merely by stored energy. To illustrate this peculiarity more, let us consider $\varphi(e, c)=\frac{1}{2} \mathbb{C}(e-E c):(e-E c)$ and write formally the underlying operator when ignoring the boundary condition, i.e.

$$
\left(\begin{array}{c}
u \\
c
\end{array}\right) \mapsto\left(\begin{array}{c}
-\operatorname{div}(\mathbb{C}(e(u)-E c)) \\
-\Delta(\mathbb{C} E:(E c-e(u)))
\end{array}\right)
$$

This linear operator is obviously nonsymmetric (thus does not have any potential) and nonmonotone (and even not pseudomonotone) due to the 3rd-order term $-\Delta \mathbb{C} E: e(u)$. Therefore, standard methods does not seem to be applicable. Yet, advantageously, the variational structure of the static problems in Section 3 can be combined with a carefully constructed fixed point.

Proposition 4.1 (Existence of steady states). Let $\varphi: \mathbb{R}_{\mathrm{sym}}^{d \times d} \times \mathbb{R} \rightarrow \mathbb{R} \cup\{\infty\}$ be lower semicontinuous, strictly convex and coercive in the sense that $\varphi(e, c) \geq \epsilon|e|^{2}+\epsilon|c|^{q}$ for some $q>2^{* \prime}$ and $\epsilon>0$, $\mathbb{M}: \mathbb{R} \rightarrow \mathbb{R}^{d \times d}$ is continuous, bounded, and uniformly positive definite, $\varepsilon \in L^{\infty}\left(\mathbb{R}^{d}\right)$ have a positive infimum, $\alpha \geq 0$ with $\alpha>0$ on a positive-measure part of $\Gamma$, meas ${ }_{d-1}\left(\Gamma_{\mathrm{D}}\right)>0, f \in L^{2^{* 1}}\left(\Omega ; \mathbb{R}^{d}\right)$, $g \in L^{2^{\sharp \prime}}\left(\Gamma_{\mathrm{N}} ; \mathbb{R}^{d}\right)$, and $\mu_{\mathrm{ext}} \in L^{2^{\sharp \prime}}(\Gamma)$. Then the boundary-value problem (1) with boundary conditions (2) possesses at least one variational solution $(u, c) \in H_{\mathrm{D}}^{1}\left(\Omega ; \mathbb{R}^{d}\right) \times L^{q}(\Omega)$ with the corresponding chemical potential $\mu \in H^{1}(\Omega) \cap L^{\infty}(\Omega)$.

Proof. We construct the single-valued mapping $\tilde{\mu} \mapsto(u, c) \mapsto \mu$ for which the Schauder fixed-point theorem will be used. First, fixing $\tilde{\mu} \in H^{1}(\Omega)$, we solve

$$
\left.\begin{array}{ll}
\text { Minimize } & (u, c) \mapsto \int_{\Omega} \varphi(e(u), c)-\tilde{\mu} c-f \cdot u \mathrm{~d} x-\int_{\Gamma_{\mathrm{N}}} g \cdot u \mathrm{~d} S \\
\text { subject to } & u \in H_{\mathrm{D}}^{1}\left(\Omega ; \mathbb{R}^{d}\right) \text { and } c \in L^{q}(\Omega) .
\end{array}\right\}
$$

Note that the term $\tilde{\mu} c$ is integrable and $(\tilde{\mu}, c) \mapsto \tilde{\mu} c: H^{1}(\Omega) \times L^{q}(\Omega) \rightarrow L^{1}(\Omega)$ is (weak,weak)continuous due to the condition $q>2^{* \prime}$ and the Rellich theorem. Due to the assumed strict convexity of $\varphi$, this problem has a unique solution $(u, c)$. It is also important that this solution depends continuously on $\tilde{\mu}$ in the sense that $\tilde{\mu} \mapsto(u, c): H^{1}(\Omega) \rightarrow H_{\mathrm{D}}^{1}\left(\Omega ; \mathbb{R}^{d}\right) \times L^{q}(\Omega)$ is (weak,strong)-continuous, which can be seen when exploiting the assumed strict convexity of $\varphi$, cf. [15].

Having $c \in L^{1}(\Omega)$, we then

$$
\left.\begin{array}{ll}
\text { Minimize } & \mu \mapsto \int_{\Omega} \frac{1}{2} \mathbb{M}(c) \nabla \mu \cdot \nabla \mu \mathrm{d} x+\int_{\Gamma_{\mathrm{N}}} \frac{\alpha}{2} \mu^{2}-\alpha \mu_{\text {ext }} \mu \mathrm{d} S \\
\text { subject to } & \mu \in H^{1}(\Omega) .
\end{array}\right\}
$$

Due to the assumed positive definiteness of $\mathbb{M}$ and the (partial) positivity of $\alpha$, the problem (15) has a unique solution $\mu$. It is important that the mapping $c \mapsto \mu: L^{1}(\Omega) \rightarrow H^{1}(\Omega)$ is (strong,weak)continuous. Actually, even (strong,strong)-continuity can easily be proved but it is not needed for our fixed-point argument.

It should be emphasized that $\mu$ from (15) does not need to be a chemical potential corresponding to $(u, c)$. Yet, we will show that it is if $\mu=\tilde{\mu}$. Such pair $(\mu, \tilde{\mu})$ does exists due to the Schauder fixed point theorem. Here we also used that the solution $\mu$ ranges an a-priori bounded set in $H^{1}(\Omega)$ because $\mathbb{M}(\cdot)$ is assumed uniformly positive definite.

The 1st-order optimality conditions for (14) compose from the partial Gâteaux derivatives with respect to $u$ and to $c$ to vanish. The former condition means the Euler-Lagrange equation representing the weak formulation of the boundary-value problem:

$$
\begin{array}{ll}
\operatorname{div} \partial_{e} \varphi(e(u), c)+f=0 & \text { on } \Omega, \\
u=0 \text { on } \Gamma_{\mathrm{D}} \text { and } \sigma \vec{n}=g & \text { on } \Gamma_{\mathrm{N}},
\end{array}
$$

while the latter conditions written for $\tilde{\mu}=\mu$ yields

$$
\partial_{c} \varphi(e(u), c)-\mu \ni 0 \quad \text { on } \Omega .
$$


The 1st-order optimality conditions for (15) means the Euler-Lagrange equation representing the weak formulation of the boundary-value problem:

$$
\begin{array}{ll}
\operatorname{div}(\mathbb{M}(c) \nabla \mu)=0 & \text { on } \Omega, \\
\mathbb{M}(c) \nabla \mu \cdot \vec{n}+\alpha \mu=\alpha \mu_{\text {ext }} & \text { on } \Gamma .
\end{array}
$$

Altogether, (16)-(18) reveal that $(u, c)$ solves the problem (1)-(2).

Let us now investigate the steady-state variant of the static electrically-charged multi-component problem (11). This results to

$$
\begin{array}{ll}
\operatorname{div} \partial_{e} \varphi(e(u), c)+f=z_{\mathrm{DOP}} \nabla \phi & \text { on } \Omega, \\
\operatorname{div}(\mathbb{M}(c) \nabla \mu)+r(c)=0 \quad \text { with } \mu \in \partial_{c} \varphi(e(u), c)+z \phi & \text { on } \Omega, \\
\operatorname{div}(\varepsilon \nabla \phi)+z \cdot c=z_{\mathrm{DOP}}+\operatorname{div}\left(z_{\mathrm{DOP}} u\right) & \text { on } \mathbb{R}^{d},
\end{array}
$$

with $r=r(c)$ the rate of chemical reactions, to be completed by the boundary conditions (2) and $\phi(\infty)=0$.

Proposition 4.2. Let again $\varphi: \mathbb{R}_{\mathrm{sym}}^{d \times d} \times \mathbb{R}^{N} \rightarrow \mathbb{R} \cup\{\infty\}$ be lower semicontinuous, strictly convex, and now even uniformly convex in $c$ and coercive in the sense that, for some $\epsilon>0$,

$$
\begin{gathered}
\forall\left(e_{1}, c_{1}\right),\left(e_{2}, c_{2}\right) \in \mathbb{R}_{\mathrm{sym}}^{d \times d} \times \mathbb{R}^{N} \forall m_{1} \in \partial_{c} \varphi\left(e_{1}, c_{1}\right), m_{2} \in \partial_{c} \varphi\left(e_{2}, c_{2}\right): \\
\epsilon\left(\left|c_{1}\right|^{q-2} c_{1}-\left|c_{2}\right|^{q-2} c_{2}\right)\left(c_{1}-c_{2}\right) \leq\left(\partial_{e} \varphi\left(e_{1}, c_{1}\right)-\partial_{e} \varphi\left(e_{2}, c_{2}\right)\right):\left(e_{1}-e_{2}\right) \\
\quad+\left(m_{1}-m_{2}\right) \cdot\left(c_{1}-c_{2}\right), \\
\exists q>2^{* \prime} \forall(e, c) \in \mathbb{R}_{\mathrm{sym}}^{d \times d} \times \mathbb{R}^{N}: \quad \varphi(e, c) \geq \epsilon|e|^{2}+\epsilon|c|^{q} .
\end{gathered}
$$

Let further $\mathbb{M}: \mathbb{R}^{N} \rightarrow \mathbb{R}^{d \times d \times N}$ be continuous, bounded, and uniformly positive definite, $r: \mathbb{R}^{N} \rightarrow$ $\mathbb{R}^{N}$ continuous and bounded, $\varepsilon \in L^{\infty}\left(\mathbb{R}^{d}\right)$ have a positive infimum, $\alpha \geq 0$ with $\alpha>0$ on a positive-measure part of $\Gamma, z_{\mathrm{DOP}} \in L^{\infty}(\Omega), z \in L^{\infty}\left(\Omega ; \mathbb{R}^{N}\right)$, meas ${ }_{d-1}\left(\Gamma_{\mathrm{D}}\right)>0, f \in L^{2^{* \prime}}\left(\Omega ; \mathbb{R}^{d}\right)$, $g \in L^{2^{\sharp^{\prime}}}\left(\Gamma_{\mathrm{N}} ; \mathbb{R}^{d}\right)$, and $\mu_{\mathrm{ext}} \in L^{2^{\sharp^{\prime}}}\left(\Omega ; \mathbb{R}^{N}\right)$. Then the boundary-value problem (19) with boundary conditions (2) and $\phi(\infty)=0$ possesses at least one variational solution $(u, c) \in H_{\mathrm{D}}^{1}\left(\Omega ; \mathbb{R}^{d}\right) \times$ $L^{q}\left(\Omega ; \mathbb{R}^{N}\right)$ with the corresponding electrochemical potential $\mu \in H^{1}\left(\Omega ; \mathbb{R}^{N}\right)$.

Proof. We construct the single-valued mapping $\tilde{\mu} \mapsto(u, c, \phi) \mapsto \mu$ for which the Schauder fixed-point theorem will be used. First, we fix $\tilde{\mu} \in H^{1}\left(\Omega ; \mathbb{R}^{N}\right)$ and, being motivated by (12), we modify (14) as

$$
\left.\begin{array}{cc}
\operatorname{Min} / \max \quad(u, c, \phi) \mapsto & \int_{\Omega}\left(\varphi(e(u), c)+\left(z \cdot c-z_{\mathrm{DOP}}\right) \phi+z_{\mathrm{DOP}} \nabla \phi \cdot u\right. \\
& -\tilde{\mu} \cdot c-f \cdot u) \mathrm{d} x-\int_{\mathbb{R}^{d}} \frac{\varepsilon}{2}|\nabla \phi|^{2} \mathrm{~d} x-\int_{\Gamma_{\mathrm{N}}} g \cdot u \mathrm{~d} S, \\
\text { subject to } \quad u \in H_{\mathrm{D}}^{1}\left(\Omega ; \mathbb{R}^{d}\right), \quad c \in L^{q}\left(\Omega ; \mathbb{R}^{N}\right), \quad \phi \in H^{1}\left(\mathbb{R}^{d}\right) .
\end{array}\right\}
$$

Due to the assumed strict convexity of $\varphi$, this problem has a unique solution $(u, c, \phi)$ which depends continuously on $\tilde{\mu}$ in the sense that $\tilde{\mu} \mapsto(u, c, \phi): H^{1}\left(\Omega ; \mathbb{R}^{N}\right) \rightarrow H_{\mathrm{D}}^{1}\left(\Omega ; \mathbb{R}^{d}\right) \times L^{q}\left(\Omega ; \mathbb{R}^{N}\right) \times$ $H^{1}\left(\mathbb{R}^{d}\right)$ is (weak,strong)-continuous. More in detail, the existence of this saddle point is the classical (Banach-space-valued extension [4] of) von Neumann theorem [16]. The uniqueness can be proved by analyzing the optimality conditions (19a) and (19c) together with $\partial_{c} \varphi(e(u), c)+z \phi \ni \tilde{\mu}$ written as

$$
m+z \phi=\tilde{\mu} \quad \text { for some } m \in \partial_{c} \varphi(e(u), c),
$$

considered for two solutions $\left(u_{i}, c_{i}, \phi_{i}, m_{i}\right), i=1,2$, and subtracted. Using the abbreviation $u_{12}=$ $u_{1}-u_{2}, c_{12}=c_{1}-c_{2}, \phi_{12}=\phi_{1}-\phi_{2}$, and $m_{12}=m_{1}-m_{2}$, this results to the system

$$
\begin{array}{ll}
\operatorname{div}\left(\partial_{e} \varphi\left(e\left(u_{1}\right), c_{1}\right)-\partial_{c} \varphi\left(e\left(u_{2}\right), c_{2}\right)\right)=z_{\mathrm{DOP}} \nabla \phi_{12} & \text { on } \Omega, \\
\operatorname{div}\left(\varepsilon \nabla \phi_{12}\right)+z \cdot c_{12}=\operatorname{div}\left(z_{\mathrm{DOP}} u_{12}\right) & \text { on } \mathbb{R}^{d}, \\
m_{12}+z \phi_{12}=0 & \text { on } \Omega,
\end{array}
$$


with the homogeneous boundary conditions for $(23 \mathrm{a})$, i.e. $u_{12}=0$ on $\Gamma_{\mathrm{D}}$ and $\left(\partial_{e} \varphi\left(e\left(u_{1}\right), c_{1}\right)-\right.$ $\left.\partial_{e} \varphi\left(e\left(u_{2}\right), c_{2}\right)\right) \vec{n}=0$ on $\Gamma_{\mathrm{N}}$. Testing (23a) by $u_{12}$ and also (23c) by $c_{12}$ (integrated it over $\Omega$ ) and (23b) by $\phi_{12}$ (integrated it over $\mathbb{R}^{d}$ ) gives

$$
\begin{gathered}
\int_{\Omega}\left(\partial_{e} \varphi\left(e\left(u_{1}\right), c_{1}\right)-\partial_{e} \varphi\left(e\left(u_{2}\right), c_{2}\right)\right): e\left(u_{12}\right)+m_{12} \cdot c_{12} \mathrm{~d} x+\int_{\mathbb{R}^{d}} \varepsilon\left|\nabla \phi_{12}\right|^{2} \mathrm{~d} x \\
=\int_{\Omega}-z_{\mathrm{DOP}} \nabla \phi_{12} \cdot u_{12}-z \cdot c_{12} \phi_{12} \mathrm{~d} x+\int_{\mathbb{R}^{d}} z \cdot c_{12} \phi_{12}-\operatorname{div}\left(z_{\mathrm{DOP}} u_{12}\right) \phi_{12} \mathrm{~d} x \\
=\int_{\mathbb{R}^{d}}-z_{\mathrm{DOP}} \nabla \phi_{12} \cdot u_{12}-z \cdot c_{12} \phi_{12}+z \cdot c_{12} \phi_{12}-\operatorname{div}\left(z_{\mathrm{DOP}} u_{12}\right) \phi_{12} \mathrm{~d} x \\
=\int_{\mathbb{R}^{d}}-\operatorname{div}\left(z_{\mathrm{DOP}} u_{12} \phi_{12}\right) \mathrm{d} x=-\left[z_{\mathrm{DOP}} u_{12} \phi_{12}\right](\infty)=0 .
\end{gathered}
$$

We used that $z=0$ on $\mathbb{R}^{d} \backslash \Omega$ and then cancellation of the terms $\pm z \cdot c_{12} \phi_{12}$ as well as that $\phi_{12}(\infty)=0$ and $z_{\mathrm{DOP}}(\infty)=0$.

From the strict monotonicity of $\partial \varphi$, we can easily see uniqueness of the saddle point of (21), needed for the Schauder fixed point. Moreover, the mentioned continuity is to be proved by taking two right-hand sides $\tilde{\mu}_{i}, i=1,2$, in (22). This gives rise the additional term $\int_{\Omega} \tilde{\mu}_{12} \cdot c_{12} \mathrm{~d} x$ on the righthand side of (24), which can be estimated by using the Hölder inequality and then, by the assumption (20a) and the uniform convexity of the $L^{q}\left(\Omega ; \mathbb{R}^{N}\right)$-space, again obtain the desired (weak,strong)continuity of $\tilde{\mu} \mapsto c: H^{1}\left(\Omega ; \mathbb{R}^{N}\right) \rightarrow L^{q}\left(\Omega ; \mathbb{R}^{N}\right)$; cf. e.g. [10, Chap. 2].

Having $c \in L^{1}\left(\Omega ; \mathbb{R}^{N}\right)$, we again solve (15) now in addition with a term $-r(c) \cdot \mu$ and, as in the proof of Proposition 4.1, we prove that the mapping $c \mapsto \mu: L^{1}\left(\Omega ; \mathbb{R}^{N}\right) \rightarrow H^{1}\left(\Omega ; \mathbb{R}^{N}\right)$ is (strong,weak)-continuous.

It should be emphasized that $\mu$ from (15) does not need to be a chemical potential corresponding to $(u, c)$. Yet, it is if $\mu=\tilde{\mu}$. Such pair $(\mu, \tilde{\mu})$ does exist due to the Schauder fixed point theorem. Here we also used that the solution $\mu$ ranges in an a-priori bounded subset of $H^{1}(\Omega)$ because of the assumed uniform positive definiteness of $\mathbb{M}(\cdot)$ and the boundedness of the reaction rates $r(\cdot)$.

\section{Anisothermal problems}

We already mentioned that the diffusion equation (1b) or (19b) is related to the dissipation rather than the stored energy. Thermodynamically, the dissipation rate (i.e. here $\mathbb{M} \nabla \mu \cdot \nabla \mu$ ) leads to the heat production which might substantially influence temperature if the specimen is large or/and the produced heat cannot be transferred away sufficiently fast. In turn, variation of temperature may influence the dissipation mechanism and the stored energy too, and thus gives rise to a thermomechanically coupled system.

The free energy $\varphi$ as well as the mobility tensor $\mathbb{M}$ now may depend on temperature, let us denote it by $\theta$. The original system (1) then augments as

$$
\begin{array}{lrl}
\operatorname{div} \sigma+f=0 & \text { with } & \sigma=\partial_{e} \varphi(e(u), c, \theta), \\
\operatorname{div}(\mathbb{M}(c, \theta) \nabla \mu)=0 & \text { with } & \mu \in \partial_{c} \varphi(e(u), c, \theta), \\
\operatorname{div}(\mathbb{K}(c, \theta) \nabla \theta)+\mathbb{M}(c, \theta) \nabla \mu \cdot \nabla \mu=0 &
\end{array}
$$

to be solved on a bounded Lipschitz domain $\Omega \subset \mathbb{R}^{d}$. Note that (25c) involves the Fourier law, saying that the heat flux equals $-\mathbb{K}(c, \theta) \nabla \theta$. This system should be completed by suitable boundary conditions, e.g.

$$
\begin{aligned}
& u=0 \text { on } \Gamma_{\mathrm{D}}, \quad \sigma \vec{n}=g \quad \text { on } \Gamma_{\mathrm{N}}, \\
& \mathbb{M}(c, \theta) \nabla \mu \cdot \vec{n}+\alpha \mu=\alpha \mu_{\text {ext }} \text { on } \Gamma, \\
& \mathbb{K}(c, \theta) \nabla \theta \cdot \vec{n}+\gamma \theta=\gamma \theta_{\text {ext }} \text { on } \Gamma .
\end{aligned}
$$

In (25c) and (26c), $\mathbb{K}=\mathbb{K}(c, \theta)$ denotes a heat-conductivity tensor. 
In this scalar case, an interesting transformation (used also in a steady-state thermistor problem [10, Sect. 6.4]) is based on the formula $\operatorname{div}(a v)=a \operatorname{div} v+\nabla a \cdot v$. One can indeed rely on

$$
\operatorname{div}(\mu \mathbb{M}(c, \theta) \nabla \mu)=\mu \underbrace{\operatorname{div}(\mathbb{M}(c, \theta) \nabla \mu)}_{=0 \text { by }(25 \mathrm{~b})}+\mathbb{M}(c, \theta) \nabla \mu \cdot \nabla \mu=\mathbb{M}(c, \theta) \nabla \mu \cdot \nabla \mu \text {. }
$$

If $\mu \in L^{\infty}(\Omega)$, it indeed can be tested by functions from $H^{1}(\Omega)$ and thus lives in $H^{1}(\Omega)^{*}$. In the scalar case, one has the information $\mu \in L^{\infty}(\Omega)$ at disposal due to the maximum principle if the external chemical potential $\mu_{\text {ext }}$ is in $L^{\infty}(\Gamma)$. Thus, instead of $(25 \mathrm{c})$, one can equivalently consider

$$
\operatorname{div}(\mathbb{K}(c, \theta) \nabla \theta+\mu \mathbb{M}(c, \theta) \nabla \mu)=0 .
$$

Proposition 5.1 (Existence of steady states). Let $\varphi: \mathbb{R}_{\mathrm{sym}}^{d \times d} \times \mathbb{R} \times \mathbb{R} \rightarrow \mathbb{R} \cup\{\infty\}$ be lower semicontinuous and coercive in the sense that $\varphi(e, c, \theta) \geq \epsilon|e|^{2}+\epsilon|c|^{q}$ for some $q>2^{* \prime}$ and $\epsilon>0$ and $\varphi(\cdot, \cdot, \theta)$ be strictly convex for any $\theta \in \mathbb{R}$ and $\theta \mapsto \varphi(\cdot, \cdot, \theta)$ be continuous in the sense of $\Gamma$-convergence as stated in (30) below, and let $\mathbb{M}, \mathbb{K}: \mathbb{R}^{2} \rightarrow \mathbb{R}^{d \times d}$ be continuous, bounded, and uniformly positive definite, $\gamma \geq 0$, $\mu_{\mathrm{ext}} \in L^{\infty}(\Gamma), \theta_{\mathrm{ext}} \in L^{2^{\prime}}(\Gamma)$, and $\alpha$, $f$, and $g$ be as in Proposition 4.1. Then the boundary-value problem (25)-(26) possesses at least one variational solution $(u, c, \theta) \in H_{\mathrm{D}}^{1}\left(\Omega ; \mathbb{R}^{d}\right) \times L^{q}(\Omega) \times H^{1}(\Omega)$ with the corresponding chemical potential $\mu \in H^{1}(\Omega) \cap L^{\infty}(\Omega)$.

Proof. We construct the single-valued mapping $(\tilde{\mu}, \tilde{\theta}) \mapsto(u, c, \theta) \mapsto \mu$ for which the Schauder fixedpoint theorem will be used. First, fixing $\tilde{\mu} \in H^{1}(\Omega) \cap L^{\infty}(\Omega)$ and $\tilde{\theta} \in H^{1}(\Omega)$, we solve

$$
\left.\begin{array}{ll}
\text { Minimize } & (u, c) \mapsto \int_{\Omega} \varphi(e(u), c, \tilde{\theta})-\tilde{\mu} c-f \cdot u \mathrm{~d} x-\int_{\Gamma_{\mathrm{N}}} g \cdot u \mathrm{~d} S \\
\text { subject to } & u \in H_{\mathrm{D}}^{1}\left(\Omega ; \mathbb{R}^{d}\right) \text { and } c \in L^{q}(\Omega) .
\end{array}\right\}
$$

Due to the assumed strict convexity of $\varphi$, this problem has a unique solution $(u, c)$. It is also important that this solution depends continuously on $(\tilde{\mu}, \tilde{\theta})$ in the sense that $(\tilde{\mu}, \tilde{\theta}) \mapsto(u, c): H^{1}(\Omega)^{2} \rightarrow$ $H_{\mathrm{D}}^{1}\left(\Omega ; \mathbb{R}^{d}\right) \times L^{q}(\Omega)$ is (weak,strong)-continuous. In particular, we use the assumed $\Gamma$-convergence, meaning that the set-valued mapping

$$
\theta \mapsto \operatorname{epi} \varphi(\cdot, \cdot, \theta):=\left\{(e, c, a) \in \mathbb{R}_{\mathrm{sym}}^{d \times d} \times \mathbb{R}^{N} \times(\mathbb{R} \cup\{\infty\}) ; \varphi(e, c, \theta) \leq a\right\}
$$

is continuous in the Hausdorff sense, and also the Rellich compactness theorem so that the functional

$$
H_{\mathrm{D}}^{1}\left(\Omega ; \mathbb{R}^{d}\right) \times L^{q}(\Omega) \rightarrow \mathbb{R} \cup\{\infty\}:(u, c) \mapsto \int_{\Omega} \varphi(e(u), c, \tilde{\theta})-\tilde{\mu} c \mathrm{~d} x
$$

$\Gamma$-converges if $(\tilde{\mu}, \tilde{\theta})$ converges weakly in $H^{1}(\Omega)^{2}$. From this and the strict convexity, the desired continuity of $(\tilde{\mu}, \tilde{\theta}) \mapsto(u, c)$ is seen.

Further, we solve the boundary-value problem (18) now with $\mathbb{M}(c, \tilde{\theta})$ instead of $\mathbb{M}(c)$. In addition, assuming $\mu_{\text {ext }} \in L^{\infty}(\Gamma)$, we can use the maximum principle yielding the estimate ess inf $\mu_{\text {ext }}(\Gamma) \leq$ $\mu(x) \leq \operatorname{ess} \sup \mu_{\text {ext }}(\Gamma)$ for a.a. $x \in \Omega$. This estimate is independent of $(\tilde{\mu}, \tilde{\theta})$, as well as the estimates $\|\mu\|_{H^{1}(\Omega)} \leq C$ and $\|\theta\|_{H^{1}(\Omega)} \leq C$ provided $C$ is large enough.

Eventually, having $c, \mu$, and $\tilde{\theta}$ at disposal, we solve

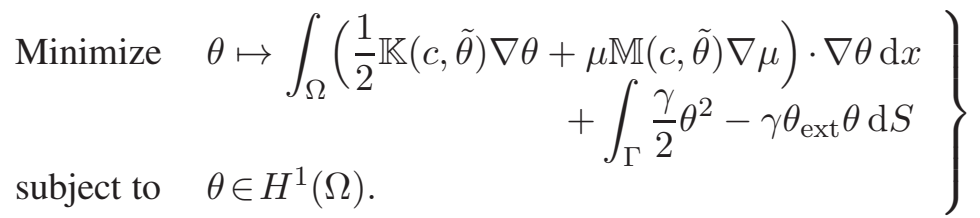


For the (strong $\times$ strong $\times$ weak,weak)-continuity of the mapping $(c, \tilde{\theta}, \tilde{\mu}) \mapsto \theta$, it is important that the weak convergence of $\mu$ in $H^{1}(\Omega)$ implies that $\operatorname{div}(\mu \mathbb{M}(c, \tilde{\theta}) \nabla \mu)$ converges weakly in $H^{1}(\Omega)^{*}$ so that the weak convergence temperatures in $H^{1}(\Omega)$ easily follows.

This allows to execute the Schauder fixed point for the mapping $(\tilde{\mu}, \tilde{\theta}) \mapsto(\mu, \theta): H^{1}(\Omega)^{2} \rightarrow$ $H^{1}(\Omega)^{2}$ in the weak topology. (In fact, even strong convergence of $\mu$ 's solving (18) now with $\mathbb{M}=$ $\mathbb{M}(c, \tilde{\theta})$ and thus also of $\theta$ 's solving (28) with $\mathbb{K}=\mathbb{K}(c, \tilde{\theta})$ and $\mathbb{M}=\mathbb{M}(c, \tilde{\theta})$ can be proved but, in contrast with the proof of Proposition 5.2, we will not need it here.)

The thermodynamical completion of the electrically-charged multicomponent system combines (19) with (25), resulting to

$$
\begin{array}{ll}
\operatorname{div} \partial_{e} \varphi(e(u), c, \theta)+f=z_{\mathrm{DOP}} \nabla \phi & \text { on } \Omega, \\
\operatorname{div}(\mathbb{M}(c, \theta) \nabla \mu)+r(c, \theta)=0 \quad \text { with } \mu \in \partial_{c} \varphi(e(u), c, \theta)+z \phi & \text { on } \Omega, \\
\operatorname{div}(\mathbb{K}(c, \theta) \nabla \theta)+\mathbb{M}(c, \theta) \nabla \mu: \nabla \mu+h(c, \theta)=\mu \cdot r(c, \theta) & \text { on } \Omega, \\
\operatorname{div}(\varepsilon \nabla \phi)+z \cdot c=z_{\mathrm{DOP}}+\operatorname{div}\left(z_{\mathrm{DOP}} u\right) & \text { on } \mathbb{R}^{d} .
\end{array}
$$

The right-hand side $\mu \cdot r(c, \theta)$ of (32c) represents the (negative) heat production where $h=h(c, \theta)$ denotes the heat-production rate due to chemical reactions. Exploiting (32b), the calculus (27) modifies to $\operatorname{div}(\mu \mathbb{M}(c, \theta) \nabla \mu)=\mathbb{M}(c, \theta) \nabla \mu: \nabla \mu-\mu \cdot r(c, \theta)$ so that the heat-transfer problem (32c) turns again to (28). The multi-component system (32) is more complicated than (25) because the maximum principle for $\mu$ and the variational structure for the heat-transfer equation (even if transformed into (28)) is not at disposal, however.

Proposition 5.2. Let $\varphi: \mathbb{R}_{\mathrm{sym}}^{d \times d} \times \mathbb{R}^{N} \times \mathbb{R} \rightarrow \mathbb{R} \cup\{\infty\}$ be lower semicontinuous and coercive in the sense that $\varphi(e, c, \theta) \geq \epsilon|e|^{2}+\epsilon|c|^{q}$ for some $q>2^{* \prime}$ and $\epsilon>0$ and $\varphi(\cdot, \cdot, \theta)$ be strictly convex satisfying (20a) uniformly for any $\theta \in \mathbb{R}$ and $\theta \mapsto \varphi(\cdot, \cdot, \theta)$ be continuous in the sense of $\Gamma$ convergence as stated previously in (30), and let $\mathbb{M}: \mathbb{R}^{N} \times \mathbb{R} \rightarrow \mathbb{R}^{d \times d \times N}$ and $\mathbb{K}: \mathbb{R}^{N} \times \mathbb{R} \rightarrow \mathbb{R}^{d \times d}$ be continuous, bounded, and uniformly positive definite, $h: \mathbb{R}^{N} \times \mathbb{R} \rightarrow \mathbb{R}$ be continuous and bounded, $\gamma \geq 0$, and $\theta_{\mathrm{ext}} \in L^{1}(\Gamma)$. Moreover, let $\mu_{\mathrm{ext}}, \varepsilon, \alpha, z_{\mathrm{DOP}}, z, f$, and $g$ be as in Proposition 4.2. Then the boundary-value problem (32) with boundary conditions (2) and $\phi(\infty)=0$ possesses at least one variational solution $(u, c, \theta, \phi) \in H_{\mathrm{D}}^{1}\left(\Omega ; \mathbb{R}^{d}\right) \times L^{q}\left(\Omega ; \mathbb{R}^{N}\right) \times W^{1, p}(\Omega) \times H^{1}\left(\mathbb{R}^{d}\right)$ with any $1 \leq p<d^{\prime}$ with the corresponding electrochemical potential $\mu \in H^{1}\left(\Omega ; \mathbb{R}^{N}\right)$.

Sketch of the proof. We organize the Schauder fixed point for a composed single-valued mapping

$$
\begin{aligned}
& (\tilde{\mu}, \tilde{\theta}) \mapsto(u, c, \phi): H^{1}\left(\Omega ; \mathbb{R}^{N}\right) \times W^{1, p}(\Omega) \rightarrow H_{\mathrm{D}}^{1}\left(\Omega ; \mathbb{R}^{d}\right) \times L^{q}\left(\Omega ; \mathbb{R}^{N}\right) \times H^{1}\left(\mathbb{R}^{d}\right), \\
& (c, \tilde{\theta}) \mapsto \mu: L^{q}\left(\Omega ; \mathbb{R}^{N}\right) \times W^{1, p}(\Omega) \rightarrow H^{1}\left(\Omega ; \mathbb{R}^{N}\right), \text { and eventually } \\
& (c, \tilde{\theta}, \mu) \mapsto \theta: L^{q}\left(\Omega ; \mathbb{R}^{N}\right) \times W^{1, p}(\Omega) \times H^{1}\left(\Omega ; \mathbb{R}^{N}\right) \rightarrow W^{1, p}(\Omega) .
\end{aligned}
$$

For (33a), the minimization variational problem (29) in the previous proof is to be replaced by the saddle-point problem (21) but now with $\varphi=\varphi(\cdot, \cdot, \tilde{\theta})$. The uniqueness of its solution is again due to (23)-(24) but now with $\varphi\left(e\left(u_{i}\right), c_{i}, \tilde{\theta}\right)$ instead of $\varphi\left(e\left(u_{i}\right), c_{i}\right)$, as well as the (weak,weak $\times$ strong $\times$ weak)-continuity of (33a).

Moreover, for (33b), we solve again the minimization problem (15) with $\mathbb{M}=\mathbb{M}(c, \tilde{\mu})$ and additionally with the term $\mu \cdot r(c, \tilde{\theta})$. In contrast to the proofs of Propositions 4.1-5.1, we now need the (strong $\times$ weak,strong)-continuity of (33b), which follows standardly by the uniform convexity of the functional in (15).

Eventually, for (33c), instead of the minimization problem (31) whose infimum might be $-\infty$ because now $\tilde{\mu} \mathbb{M}(c, \tilde{\theta}) \nabla \tilde{\mu} \cdot \nabla \theta \notin L^{1}(\Omega)$ in general since the $L^{\infty}$-estimate on $\tilde{\mu}$ (and thus on $\tilde{\mu}$ too) is not at disposal, we should solve (32c) with the boundary condition (26c) with $\mathbb{K}=\mathbb{K}(c, \tilde{\theta})$, $\mathbb{M}=\mathbb{M}(c, \tilde{\theta})$, and the heat sources $h(c, \tilde{\theta})+\mu \cdot r(c, \tilde{\theta})$, by the non-variational method. By the classical Stampacchia [14] transposition method, see also e.g. [10, Section 3.2.5], this linear boundary-value problem has a unique variational solution $\theta$ which belongs to $W^{1, p}(\Omega)$ with any $1 \leq p<d^{\prime}=$ $d /(d-1)$. The (strong $\times$ weak $\times$ strong,weak)-continuity of (33c) is obvious. 
Altogether, the Schauder fixed-point relies on the weak continuity of the mapping $(\tilde{\mu}, \tilde{\theta}) \mapsto$ $(\mu, \theta): H^{1}\left(\Omega ; \mathbb{R}^{N}\right) \times W^{1, p}(\Omega) \rightarrow H^{1}\left(\Omega ; \mathbb{R}^{N}\right) \times W^{1, p}(\Omega)$.

\section{Concluding remarks}

Remark 6.1 (Uniqueness). Interestingly, it is not clear whether the solution of the static problem in Proposition 3.1 is unique, even if $\varphi$ is strictly convex so that the solution obtained in the proof of Proposition 3.1 by solving (9) is unique. An analogous comment is relevant to the problem in Proposition 3.2. As far as all steady-state but non-static problems, the nonuniqueness is rather to be expected.

Remark 6.2 (About the fixed-point strategy). Reorganizing the fixed point as $\tilde{c} \mapsto \mu \mapsto(u, c)$ or $\tilde{c} \mapsto$ $\mu \mapsto(u, c, \phi)$ and then pursuing a "construction" of $c=\tilde{c}$ would bring troubles with compactness needed in the Schauder fixed-point theorem because any information about $\nabla c$ is missing. Note that, from (6), we could think about $\nabla c=\partial_{\mu e}^{2} \varphi^{*}(e(u), \mu) \nabla e(u)+\partial_{\mu \mu}^{2} \varphi^{*}(e(u), \mu) \nabla \mu$ but it would need to have $\nabla e(u)$ estimated. Therefore, it does not seem much freedom to organize the proof of the "non-static" Propositions 4.1-5.2. A certain freedom is in (31) which may involve $\tilde{\mu}$ instead of $\mu$ because the weak convergence of $\mu$ 's is enough for (31). This freedom is not applicable to (32c) used in the proof of Proposition 5.2, however.

Remark 6.3 (Elimination of the saddle-point structure). Making maximization with respect to $\phi$ in (12) or in (21) may eliminate the $\phi$ variable, cf. also e.g. [17, Sect.49.2] for a general viewpoint. Using (11c) together with the calculus

$$
\begin{gathered}
\int_{\Omega} z \phi \cdot c-z_{\mathrm{DOP}} \phi+z_{\mathrm{DOP}} \nabla \phi \cdot u \mathrm{~d} x=\int_{\mathbb{R}^{d}} \phi\left(z \cdot c-z_{\mathrm{DOP}}-\operatorname{div}\left(z_{\mathrm{DOP}} u\right)\right) \mathrm{d} x \\
=-\int_{\mathbb{R}^{d}} \phi \operatorname{div}(\varepsilon \nabla \phi)=\int_{\mathbb{R}^{d}} \varepsilon|\nabla \phi|^{2} \mathrm{~d} x,
\end{gathered}
$$

the convex/concave problem (21) turns into the convex constrained problem:

$$
\left.\begin{array}{cl}
\text { Minimize } & (u, c, \phi) \mapsto \int_{\Omega} \varphi(e(u), c)-\tilde{\mu} \cdot c-f \cdot u \mathrm{~d} x \\
& +\int_{\mathbb{R}^{d}} \frac{\varepsilon}{2}|\nabla \phi|^{2} \mathrm{~d} x-\int_{\Gamma_{\mathrm{N}}} g \cdot u \mathrm{~d} S, \\
\text { subject to } & \operatorname{div}(\varepsilon \nabla \phi)+z \cdot c=z_{\mathrm{DOP}}+\operatorname{div}\left(z_{\mathrm{DOP}} u\right) \text { on } \mathbb{R}^{d}, \\
& u \in H_{\mathrm{D}}^{1}\left(\Omega ; \mathbb{R}^{d}\right), \quad c \in L^{1}\left(\Omega ; \mathbb{R}^{N}\right), \quad \phi \in H^{1}\left(\mathbb{R}^{d}\right) .
\end{array}\right\}
$$

Remark 6.4 (Towards electroneutrality). We can further eliminate the electrostatic potential by introducing the electrical induction $\vec{d}=\varepsilon \nabla \phi$ and, defining the Banach space

$$
\begin{aligned}
& L_{\mathrm{rot}, \varepsilon}^{2}\left(\mathbb{R}^{d} ; \mathbb{R}^{d}\right):=\left\{\vec{d} \in L^{2}\left(\mathbb{R}^{d} ; \mathbb{R}^{d}\right) ; \exists \phi \in H^{1}\left(\mathbb{R}^{d}\right):\right. \\
& \vec{d}=\varepsilon \nabla \phi \text { in the sense of distributions }\},
\end{aligned}
$$

rewrite (35) as

$$
\left.\begin{array}{ll}
\text { Minimize } & (u, c, \phi) \mapsto \int_{\Omega} \varphi(e(u), c)-\tilde{\mu} \cdot c-f \cdot u \mathrm{~d} x \\
& \quad+\int_{\mathbb{R}^{d}} \frac{1}{2 \varepsilon}|\vec{d}|^{2} \mathrm{~d} x-\int_{\Gamma_{\mathrm{N}}} g \cdot u \mathrm{~d} S, \\
\text { subject to } & \operatorname{div} \vec{d}+z \cdot c=z_{\mathrm{DOP}}+\operatorname{div}\left(z_{\mathrm{DOP}} u\right) \text { on } \mathbb{R}^{d}, \\
& u \in H_{\mathrm{D}}^{1}\left(\Omega ; \mathbb{R}^{d}\right), \quad c \in L^{1}\left(\Omega ; \mathbb{R}^{N}\right), \quad \vec{d} \in L_{\mathrm{rot}, \varepsilon}^{2}\left(\mathbb{R}^{d} ; \mathbb{R}^{d}\right) .
\end{array}\right\}
$$


It reveals an asymptotics for $\varepsilon \rightarrow 0$ : namely, assuming $\varepsilon(x)=\epsilon \varepsilon_{0}(x)$, the space $L_{\text {rot, } \varepsilon}^{2}\left(\mathbb{R}^{d} ; \mathbb{R}^{d}\right)$ is independent of $\epsilon$ and then $\|\vec{d}\|_{L^{2}\left(\mathbb{R}^{d} ; \mathbb{R}^{d}\right)}=\mathscr{O}\left(\epsilon^{1 / 2}\right)$ for $\epsilon \rightarrow 0$ and thus

$$
\left\|z \cdot c-z_{\mathrm{DOP}}-\operatorname{div}\left(z_{\mathrm{DOP}} u\right)\right\|_{H^{-1}\left(\mathbb{R}^{d} ; \mathbb{R}^{d}\right)}=\mathscr{O}\left(\epsilon^{1 / 2}\right) .
$$

In particular, in the limit, one may expect the electroneutrality, i.e. $z \cdot c-z_{\mathrm{DOP}}-\operatorname{div}\left(z_{\mathrm{DOP}} u\right)=0$. Without any rigorous justification, this ansatz is indeed often used in computational implementation if the specimen size is substantially bigger than the so-called Debye length to avoid spatially extremely stiff problems arising for small permittivities $\varepsilon$, cf. e.g. [5].

Remark 6.5 (Thermodynamics). Inspecting evolution variant of the anisothermal electricallycharged problem is illustrative. For simplicity, let us consider the special free energy suppressing thermo-mechanical interactions by assuming

$$
\psi(e, c, \theta, \vec{e})=\psi_{\mathrm{ME}}(e, c)+\psi_{\mathrm{TH}}(\theta)-\frac{\varepsilon}{2}|\vec{e}|^{2}
$$

with $\vec{e}$ standing for the intensity of the electrostatic field $\nabla \phi$. In particular, both entropy as well as internal energy separates electro-chemical-mechanical variables and the heat variable. We have

$$
\sigma=\partial_{e} \psi(e, c), \quad \eta=-\partial_{\theta} \psi(\theta), \quad \mu=\partial_{c} \psi(e, c), \quad \vec{d}=-\partial_{\vec{e}} \psi(\vec{e})
$$

with $\eta$ the entropy and $\vec{d}$ the electric induction being equal to $\varepsilon \vec{e}$. The evolution variant of the most general system (32) then reads as:

$$
\begin{aligned}
& \varrho \ddot{u}-\operatorname{div} \sigma=f-z_{\mathrm{DOP}} \nabla \phi \quad \text { with } \quad \sigma=\partial_{e} \psi_{\mathrm{ME}}(e(u), c), \\
& \dot{c}-\operatorname{div}(\mathbb{M}(c, \theta) \nabla \mu)=r(c, \theta) \quad \text { with } \quad \mu=\partial_{c} \psi_{\mathrm{ME}}(e, c)+z \phi, \\
& c_{\mathrm{v}}(\theta) \dot{\theta}-\operatorname{div}(\mathbb{K}(c, \theta) \nabla \theta)=\mathbb{M}(c, \theta) \nabla \mu: \nabla \mu+h(c, \theta)-\mu \cdot r(c, \theta), \\
& \operatorname{div}(\varepsilon \nabla \phi)+z \cdot c=z_{\mathrm{DOP}}+\operatorname{div}\left(z_{\mathrm{DOP}} u\right),
\end{aligned}
$$

with the heat capacity $c_{\mathrm{V}}(\theta)=-\theta \psi_{\mathrm{TH}}^{\prime \prime}(\theta)$, and with the boundary conditions (26). The heat production and chemical-reaction rates can be naturally assumed to vanish at $\theta=0$, i.e. $h(c, 0)=0$ and $r(c, 0)=0$, and then one can prove $\theta \geq 0$. Note that, instead of a full Maxwell electromagnetic system, we kept the electrostatic equation (39d), which reflects the well acceptable modelling assumption that the thermo-chemical-mechanical processes are much slower than the electromagnetic processes and that the electric currents are rather small so that the magnetic effects can be neglected. The balance of the electro-chemical-mechanical energy can be revealed by testing the particular equations (39a,b,d) successively by $\dot{u}, \mu$, and $\dot{\phi}$ :

$$
\begin{aligned}
\frac{\mathrm{d}}{\mathrm{d} t}\left(\int_{\Omega} \frac{\varrho}{2}|\dot{u}|^{2}\right. & \left.+\psi_{\mathrm{ME}}(e(u), c)+z \phi \cdot c-z_{\mathrm{DOP}} \phi+z_{\mathrm{DOP}} \nabla \phi \cdot u \mathrm{~d} x-\int_{\mathbb{R}^{d}} \frac{\varepsilon}{2}|\nabla \phi|^{2} \mathrm{~d} x\right) \\
& +\underbrace{\int_{\Omega} \mathbb{M}(c, \theta) \nabla \mu: \nabla \mu \mathrm{d} x+\int_{\Gamma} \alpha \mu^{2} \mathrm{~d} S}_{\text {dissipation rate }} \\
& =\int_{\Omega} f \cdot \dot{u}+r(c, \theta) \cdot \mu \mathrm{d} x+\int_{\Gamma_{\mathrm{N}}} g \cdot \dot{u} \mathrm{~d} S+\int_{\Gamma} \alpha \mu_{\mathrm{ext}} \mu \mathrm{d} S .
\end{aligned}
$$

Using again (34) and adding also (39c) tested by 1 we obtain the total-energy balance

$$
\begin{aligned}
& \frac{\mathrm{d}}{\mathrm{d} t}(\int_{\Omega} \underbrace{\frac{\varrho}{2}|\dot{u}|^{2}}_{\begin{array}{c}
\text { kinetic } \\
\text { energy }
\end{array}}+\underbrace{\psi_{\mathrm{ME}}(e(u), c)+C_{\mathrm{v}}(\theta)}_{\begin{array}{c}
\text { internal chemo-thermo- } \\
\text {-mechanical energy }
\end{array}} \mathrm{d} x+\int_{\mathbb{R}^{d}} \underbrace{\frac{\varepsilon}{2}|\nabla \phi|^{2}}_{\begin{array}{c}
\text { electrostatic } \\
\text { energy }
\end{array}} \mathrm{d} x) \\
& =\underbrace{\int_{\Gamma_{\mathrm{N}}} g \cdot \dot{u} \mathrm{~d} S+\int_{\Omega} f \cdot \dot{u}+h(c, \theta) \mathrm{d} x}_{\begin{array}{c}
\text { power of mechanical load and of } \\
\text { the heat from chemical reactions }
\end{array}}+\underbrace{\int_{\Gamma} \alpha \mu_{\text {ext }} \mu \mathrm{d} S}_{\begin{array}{c}
\text { power of } \\
\text { chemical load }
\end{array}}+\underbrace{\int_{\Gamma} \gamma\left(\theta_{\text {ext }}-\theta\right) \mathrm{d} S}_{\begin{array}{c}
\text { power of the } \\
\text { boundary heat flux }
\end{array}} .
\end{aligned}
$$


The heat part of the internal energy is $C_{\mathrm{v}}(\theta)=\psi_{\mathrm{TH}}(\theta)-\theta \psi_{\mathrm{TH}}^{\prime}(\theta)$. The analysis of the system (39) in general however does not seem clear without modification by some gradient terms, as already mentioned at the end of Section 2.

Remark 6.6 (Nonconvex free energies $\varphi$ ). Some applications call for nonconvex $\varphi$. E.g. a doublewell potential in $c$ is used in modelling of phase separation. The existence of the simplest static problem (9) obviously requires some regularization, most conventionally by adding a gradient term like $\frac{1}{2} \epsilon|\nabla c|^{2}$. Yet, the nonconvexity certainly destroys the fixed-point argument in the "non-static" Propositions 4.1-5.2. The same comment applies for nonconvexity in terms of $e$ and, in particular, arising from geometrical nonlinearity in a large-strain generalization like in [1]. The possible nonexistence of steady states may be relatively expectable due to autonomous oscillations sometimes reported in literature in the corresponding evolutionary systems, see e.g. [2,12], although strictly speaking those experimental experience rather documents nonexistence of stable steady states only.

Acknowledgement: The author is very indebted for inspiring discussions with dr. Jürgen Fuhrmann and prof. Alexander Mielke at Weierstrass Inst. Berlin about polymer-electrolyte fuel cells and about electroneutrality.

\section{References}

[1] L. Anand. A thermo-mechanically-coupled theory accounting for hydrogen diffusion and large elastic-viscoplastic deformations of metals. Intl. J. Solids Structures, 48:962-971, 2011.

[2] J. Benziger, E. Chia, J. F. Moxley, and I. G. Kevrekidis. The dynamic response of PEM fuel cells to changes in load. Chemical Engineering Science, 60:1743-1759, 2005.

[3] M. A. Biot. General theory of three-dimensional consolidation. J. Appl. Phys., 12:155-164, 1941.

[4] K. Fan. Fixed-point and minimax theorems in locally convex linear spaces. Proc. Nat. Acad. Sci. USA, 38:121126, 1952.

[5] J. Fuhrmann. Mathematical and numerical modeling of flow, transport and reactions in porous structures of electrochemical devices. In P. Bastian et al., editor, Simulation of Flow in Porous Media: Appl. in Energy and Environment. J.Wiley, to appear.

[6] M. Latroche. Structural and thermodynamic properties of metallic hydrides used for energy storage. J. Physics \& Chemistry Solids, 65:517-522, 2004.

[7] G.G. Libowitz. Metallic hydrides; fundamental properties and applications. J. Physics \& Chemistry Solids, 55:1461-1470, 1994.

[8] K. Promislow and B. Wetton. PEM fuel cells: a mathematical overview. SIAM J. Appl. Math., 70:369-409, 2009.

[9] K. R. Rajagopal. On a hierarchy of approximate models for flows of incompressible fluids through porous solids. Math. Models Meth. Appl. Sci., 17:215-252, 2007.

[10] T. Roubíček. Nonlinear Partial Differential Equations with Applications. Birkhäuser, Basel, 2nd edition, 2013.

[11] T. Roubíček and G. Tomassetti. Thermomechanics of hydrogen storage in metallic hydrides: modeling and analysis. Discr. Cont. Dyn. Syst. B, 14:2313-2333, 2014.

[12] D. G. Sanchez, D. G. Diaz, R. Hiesgen, I. Wehl, and K. A. Friedrich. Oscillations of PEM fuel cells at low cathode humidification. J. Electroanalytical Chemistry, 649:219-231, 2010.

[13] R. E. Showalter and U. Stefanelli. Diffusion in poro-elastic media. Math. Methods Appl. Sci., 27:2131-2151, 2004.

[14] G. Stampacchia. Le problème de Dirichlet pour les équations elliptiques du second ordre à coefficients discontinus. Ann. Inst. Fourier (Grenoble), 15:189-258, 1965.

[15] A. Visintin. Strong convergence results related to strict convexity. Comm. Partial Diff. Equations, 9:439-466, 1984.

[16] J. von Neumann. Zur Theorie der Gesellschaftsspiele. Math. Ann., 100:295-320, 1928.

[17] E. Zeidler. Nonlinear Functional Analysis and its Applications. I-IV. Springer, New York, 1985-90. 